\title{
Protective and therapeutic effects of Danhong injection on acute pancreatitis-associated lung injury
}

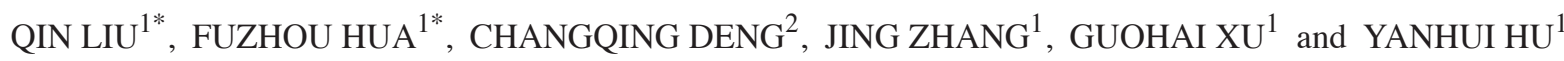 \\ ${ }^{1}$ Department of Anesthesiology, The Second Affiliated Hospital of Nanchang University, \\ Nanchang, Jiangxi 330006; ${ }^{2}$ Department of Gastroenterology, The Affiliated Hospital of Jiangxi \\ University of Traditional Chinese Medicine, Nanchang, Jiangxi 330000, P.R. China
}

Received October 13, 2016; Accepted June 2, 2017

DOI: $10.3892 / \mathrm{mmr} .2017 .7526$

\begin{abstract}
Lung functional impairment caused by acute pancreatitis (AP) is the primary contributor to AP-associated mortality. Previous studies have reported that AP-associated lung injury is associated with systemic inflammatory response syndrome and oxidative stress. In the present study, the protective effects of Danhong injection (DHI), a widely used Chinese Traditional Medicine preparation, on AP-associated lung injury in rats was examined. The myeloperoxidase activity, malondiadelhyde level and superoxide dismutase activity determination demonstrated the anti-inflammatory and anti-oxidative properties of DHI. The results of western blotting and reverse-transcription-semi-quantitative polymerase chain reaction indicated that DHI could protect rats against AP-associated lung injury, and the protective effect was associated with the suppression of nuclear factor $-\kappa \mathrm{B}$ activation and cell adhesion molecule expression, and the reduction of neutrophil infiltration and oxidative stress levels. As demonstrated by HE staining, DHI inhibited the pancreas and lung tissue injury. Therefore, DHI could be a potential candidate for the treatment of patients with AP-associated lung injury.
\end{abstract}

\section{Introduction}

Acute pancreatitis (AP) is one of the most prominent emerging diseases in the world; $15 \%$ of cases are severe AP, with an associated mortality of $\sim 10 \%$ (1). Up to $20 \%$ of all mortalities induced by AP are associated with acute lung injury, which is the predominant cause of mortality within the first week of pancreatitis (2). Previous studies indicated that AP-associated

Correspondence to: Mr. Yanhui Hu, Department of Anesthesiology, The Second Affiliated Hospital of Nanchang University, 1 Min De Road, Nanchang, Jiangxi 330006, P.R. China E-mail: yanhuihu0605@163.com

*Contributed equally

Key words: Danhong injection, acute pancreatitis, lung injury, protective effects, mechanism lung injury may be associated with systemic inflammatory response syndrome, including activation of neutrophils and macrophages and certain cytokines (3-5). Furthermore, previous research has indicated that oxidative stress resulting from an imbalance between pro-oxidants and antioxidants also serves an important role in the pathogenesis of AP-associated lung injury (6-8).

Danhong injection (DHI), a widely used Chinese Medicine preparation extracted from Salvia miltiorrhiza (Danshen in Chinese) and Carthamus tinctorius (Honghua in Chinese), had been used extensively in the clinic to treat cardiovascular diseases, such as coronary heart disease and cerebral ischemia (9-11). The main components of DHI are danshensu, protocatechuic aldehyde, savianolic acid B, rosmarinic acid and hydroxysafflor yellow A (12-14), and exerts anti-inflammatory, anti-oxidative and anti-fibrinolytic properties $(10,11,15-18)$.

In the present study, the protective effects of DHI on AP-associated lung injury were evaluated. The effects of DHI on lung and pancreas pathological changes, malondiadelhyde (MDA) level, and myeloperoxidase (MPO) and superoxide dismutase (SOD) activities were investigated. Furthermore, the influences of DHI in the expression of nuclear factor (NF)- $\kappa \mathrm{B}$ and cell adhesion molecules in lung tissues were examined. The results demonstrated the protective effects of DHI on AP-associated lung injury. The mechanism may be due to the suppression of NF- $\mathrm{NB}$ activation and cell adhesion molecule expression, and the reduction of neutrophil infiltration and oxidative stress levels.

\section{Materials and methods}

Chemical and reagents. DHI was obtained from Shangdong Buchang Pharmaceutical Co., Ltd. (Jinan, China). MPO, SOD and MDA detection kits were purchased from Nanjing Jiancheng Bionengineering Institute (Nanjing, China). Sodium taurocholate was from Sigma-Aldrich; Merck KGaA (Darmstadt, Germany). Other reagents were commercially available in China.

Animals and animal model. All experiments were performed according to the protocols approved by the Animal Care Committee of Nanchang University (Jiangxi, China). A total of 60 male Sprague-Dawley rats (4-6 weeks, 200-220 g) were 
supplied by Laboratory Animal Center of Jiangxi University of Traditional Chinese Medicine (Nanchang, China). All rats were acclimated for 7 days prior to the experiment, housed in standard shoebox cages in a climate controlled environment with an ambient temperature of $23^{\circ} \mathrm{C}$ and a 12 -h light/dark cycle, and had free access to standard laboratory food and water. The rats were maintained under controlled environmental conditions and fasted for $24 \mathrm{~h}$ with free access to water prior to experiments. AP was induced with $3 \%$ sodium taurocholate by retrograde injection into the pancreatic duct as previously described (19). Briefly, rats were anesthetized with intraperitoneal sodium pentobarbital (Sigma-Aldrich; Merck $\mathrm{KGaA}$ ) at a dose of $50 \mathrm{mg} / \mathrm{kg}$. The abdomen was opened by midline incision to allow manipulation of the duodenum and biliopancreatic duct. The common bile duct was occluded, and the duodenal wall was punctured on the antimesenteric side with a 24-gauge catheter. The catheter was advanced into the papilla vateri and fixed to the duodenal wall. For inducing AP, the catheter was brought near the pancreatic canal and $3 \%$ trichloroacetic acid (TCA, $0.1 \mathrm{ml} / 100 \mathrm{~g}$; Sigma-Aldrich; Merck KGaA) was infused slowly using a pump according to the retrograde ductal injection model, followed by closure of the abdomen in two layers. The same procedure was applied to the sham-operated group, to which $0.9 \% \mathrm{NaCl}$ was administered instead of TCA. No mortality was observed in the rats after AP was induced.

All animals were randomly assigned to the three groups ( $\mathrm{n}=20 /$ group): i) Control (N), ii) AP and iii) DHI + AP (20 rats). Each group was randomly divided into two time-dependent subgroups (A, AP group $12 \mathrm{~h}$; B, DHI + AP group $12 \mathrm{~h}$; C, AP group $24 \mathrm{~h} ; \mathrm{D}, \mathrm{DHI}+\mathrm{AP}$ group $24 \mathrm{~h}$ ) after the induction of AP. In the DHI + AP group, DHI was administered $(8 \mathrm{ml} / \mathrm{kg})$ intravenously 1 and $12 \mathrm{~h}$ after inducing $\mathrm{AP}$, and the other groups was subjected to the same amount of normal saline.

Rats were sacrificed 12 or $24 \mathrm{~h}$ after the induction of AP, and the blood samples were obtained via the retro-orbital sinus using a $1.5 \mathrm{ml}$ tube. After $30 \mathrm{~min}$ of standing, the serum was obtained by centrifugation $\left(1,500 \mathrm{x} \mathrm{g}, 15 \mathrm{~min}, 4^{\circ} \mathrm{C}\right)$ and $100 \mu \mathrm{l}$ was used to measure serum amylase activity. The left upper lung tissues were dissected for determination of the wet/dry ratio immediately. The left lower lung tissues and head of pancreas were fixed in $4 \%$ paraformaldehyde for histopathologic analysis, and then the other portions of lung and pancreatic tissues were removed and stored at $-70^{\circ} \mathrm{C}$ until use.

Determination of the wet/dry ratio of lung. After the mice were sacrificed, the left upper lung tissues $(\sim 1 \mathrm{~g})$ were dissected, cleansed of blood with absorbent paper, weighed to obtain the 'wet' weight, torrefied in an $80^{\circ} \mathrm{C}$ thermostatic baking oven for $48 \mathrm{~h}$, and weighed again to obtain the 'dry' weight. Subsequently, the ratio of wet lung to dry lung was calculated to assess tissue edema.

Pathological analysis of lung and pancreas. For pathological analysis, the lung tissues and pancreas were processed by hematoxylin and eosin (HE) staining; $\sim-\mu \mathrm{m}$ thick sections were cut and then heat fixed, deparaffinized and rehydrated through a series of xylene and graded alcohols $(100,95,85$, 75\%) and merged in distilled water. Section were stained with hematoxylin for $5 \mathrm{~min}$, washed with water, and then stained with $0.5 \%$ eosin for $1-3 \mathrm{~min}$ at room temperature. After a further wash, the sections were sealed by neutral balsam. The specimens were examined under a light microscope, and scored by two blinded pathologists with expertise in lung and pancreatic pathology. The score of the pancreas and lung were determined using Schmidt's Method (20) and Tanino Method (21), respectively.

Amylase levels, MPO activity, MDA level and SOD activity determination of the lung and pancreas. MPO activity was used as a marker of neutrophil infiltration. In addition, the level of MDA is an index of membrane lipid peroxidation, and SOD activity is an index of superoxide toxicity. Lung and pancreas tissues were frozen in liquid nitrogen and then homogenized in PBS. The amylase levels in the tissue were detected by an Amylase Assay kit (Abnova, Taipei, Taiwan). The MPO activities in their homogenates were examined using a MPO determination kit. The remaining homogenates were centrifuged at $2,000 \mathrm{xg}$ for $10 \mathrm{~min}$ at $4^{\circ} \mathrm{C}$, and the supernatants were used to detect the level of MDA and the SOD activity by using the MDA and SOD determination kits, respectively, according to the manufacturer's protocol.

Reverse-transcription-semi-quantitative polymerase chain reaction ( $R T-s q P C R$ ). Total RNA was extracted from rat lungs according using TRIzol reagent (Thermo Fisher Scientific, Inc., Waltham, MA, USA) (22). Total RNA was reverse transcribed into cDNA using a ReverTraAce (Toyobo Life Science, Osaka, Japan). The mRNA expression levels of vascular cell adhesion protein 1 (VCAM-1), intracellular adhesion molecule 1 (ICAM-1), NF- $\kappa$ B p65 and $\beta$-actin were determined using a SYBR qPCR mix (Toyobo Life Science) and the ABI PRISM 7500 Sequence Detection system (Applied Biosystems; Thermo Fisher Scientific, Inc.), using the primer sequences listed in Table I. The PCR product was detected by $1.2 \%$ agarose gel electrophoresis, and the amount of PCR product was estimated by a gel imaging system (ChemiDoc XRS Image $\mathrm{Lab}^{\mathrm{TM}}$ software version 3.0, Bio-Rad Laboratories, Inc., Hercules, CA, USA). The intensity of VCAM-1, ICAM-1 and $\mathrm{NF}-\kappa \mathrm{B}$ was normalized against $\beta$-actin content.

Western blotting. Proteins were extracted from cells using lysis buffer containing $50 \mathrm{mM}$ Tris- $\mathrm{HCl}(\mathrm{pH} 8.0), 50 \mathrm{mM} \mathrm{KCl}$, $5 \mathrm{mM}$ DTT, $1 \mathrm{mM}$ EDTA, $0.1 \%$ SDS, $0.5 \%$ Triton X-100 and protease inhibitor cocktail tablets (Roche Applied Science, Penzberg, Germany). The proteins were separated by on $10 \%$ gels by SDS-PAGE. The quantity of protein loaded onto the gels was $50 \mu \mathrm{g}$, and proteins were then transferred onto polyvinylidene fluoride membranes (EMD Millipore, Billerica, MA, USA). The membranes were probed with antibodies specific for VCAM-1 (cat no. 14694), ICAM-1 (cat no. 4915) and NF-kB p65 (cat no. 8242) (Cell Signaling Technology, Inc., Danvers, MA, USA) at a dilution of 1:1,000 and $\beta$-actin (cat no. sc-47778, Santa Cruz Biotechnology, Inc., Dallas, TX, USA) at a dilution of 1:2,000 with PBST, overnight at $4^{\circ} \mathrm{C}$. Finally, the membrane was incubated with a horseradish peroxidase conjugated secondary mouse antibody or antibody rabbit (cat nos. sc-2314 or sc-2313, Santa Cruz Biotechnology, Inc.) for $1 \mathrm{~h}$ at room temperature. Immunocomplexes were visualized with an enhanced chemiluminescence system 
Table I. Primer sequences for reverse transcription-semi-quantitative polymerase chain reaction.

\begin{tabular}{lll}
\hline Gene & \multicolumn{1}{c}{ Forward $\left(5^{\prime}-3^{\prime}\right)$} & \multicolumn{1}{c}{ Reverse $\left(5^{\prime}-3^{\prime}\right)$} \\
\hline VCAM-1 & TGGGAAGGTGAAGACAGAGG & TTGGGAATAGAATCAGTTTGGT \\
ICAM-1 & TGGGTCATAATTGTTGGTG & CAGACCAGCAGCACTCCATC \\
NF- $\mathrm{kB}$ p65 & GGCAGCACTCCTTATCAACC & GGTGTCGTCCCATCGTAG \\
$\beta$-actin & TCCTGTGGCATCCACGAAACT & GAAGCATTTGCGGTGGACGAT
\end{tabular}

VCAM-1, vascular cell adhesion protein 1; ICAM-1, intracellular adhesion molecule 1; NF- $\mathrm{B}$, nuclear factor- $\mathrm{kB}$.

A

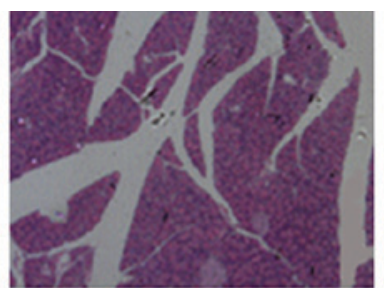

$\mathrm{D}$

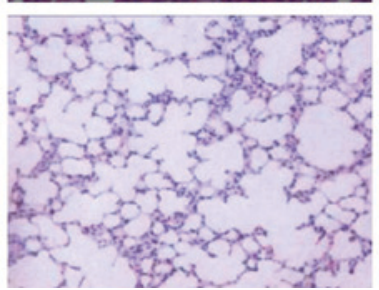

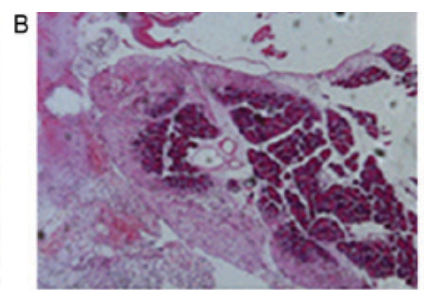
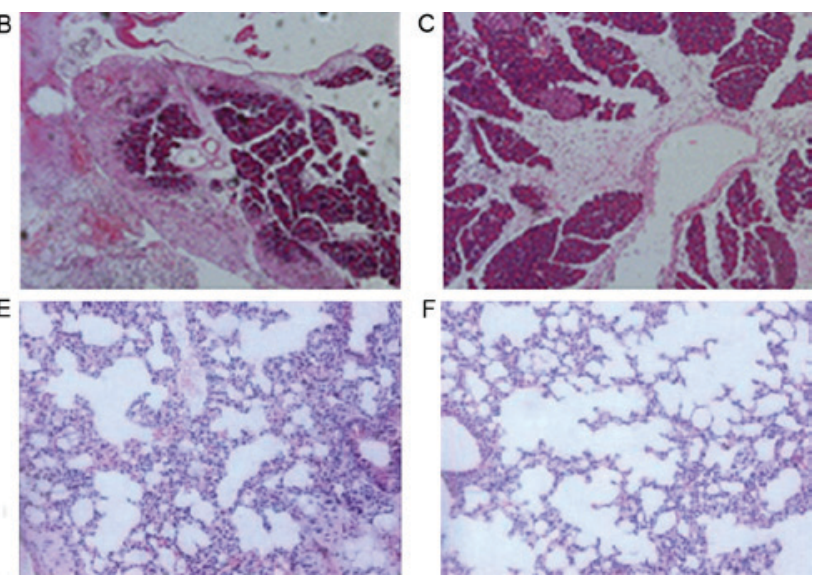

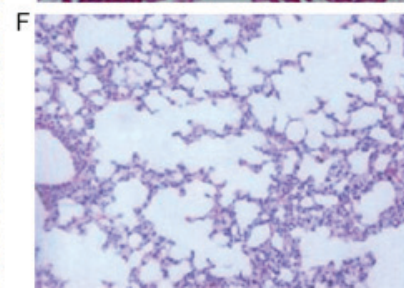

Figure 1. Pathological analysis of the pancreas and lung at $24 \mathrm{~h}$ after the induction of AP, as assessed by hematoxylin and eosin staining. Images of the pancreas in the (A) control, (B) AP and (C) DHI + AP groups. Images of the lung in the (D) control, (E) AP and (F) DHI + AP groups. Magnification, x100. AP, acute pancreatitis; DHI, Danhong injection.

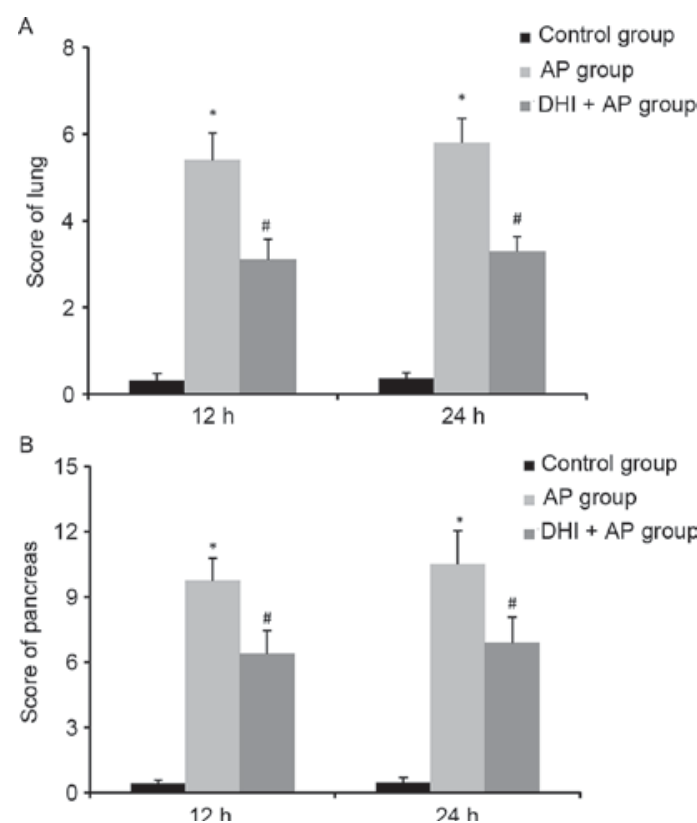

Figure 2. Histopathologic score of the (A) lung and (B) pancreas. Data are presented as the mean \pm standard error ( $\mathrm{n}=10 /$ group). ${ }^{*} \mathrm{P}<0.01$ vs. control group; ${ }^{\mathrm{P}}<0.01$ vs. AP group. AP, acute pancreatitis; DHI, Danhong injection.

(Thermo Fisher Scientific, Inc.). ImageJ software version 3.0 (National Institutes of Health, Bethesda, MD, USA) was used to compare the density of bands on the blots.

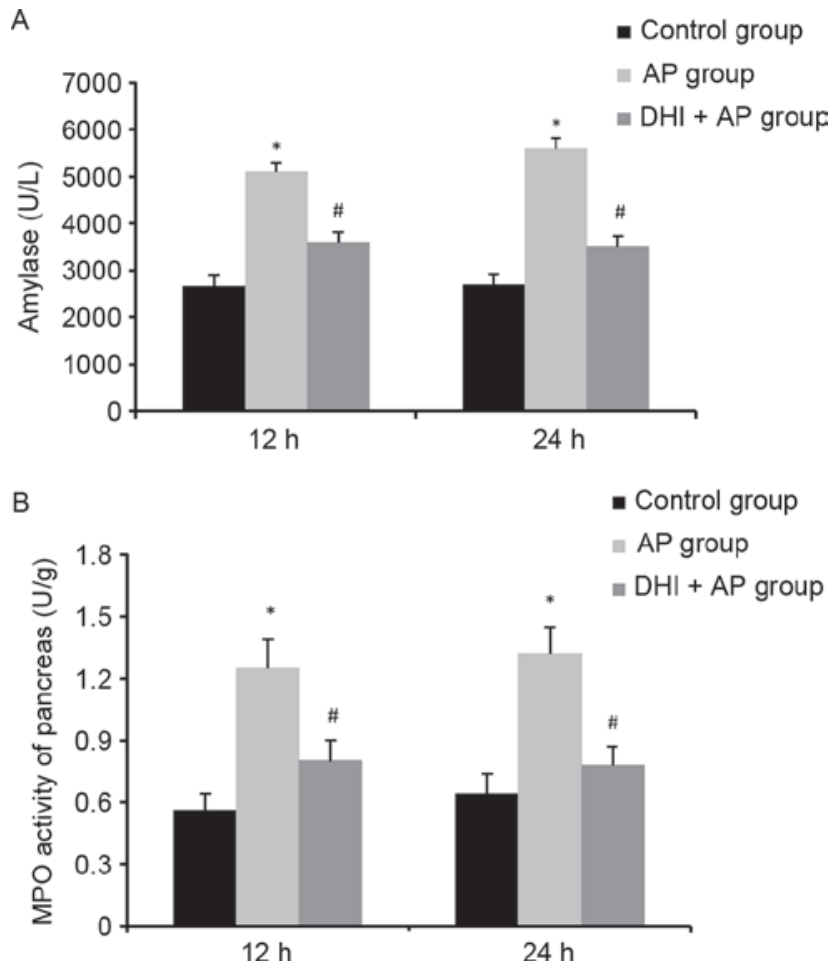

Figure 3. Effects of DHI on pancreatic injury AP rats. (A) Plasma amylase activity and (B) pancreatic MPO activity. Data are presented as the mean \pm standard error ( $\mathrm{n}=10$ /group). ${ }^{*} \mathrm{P}<0.01$ vs. control group; ${ }^{\#} \mathrm{P}<0.01$ vs. AP group. AP, acute pancreatitis; DHI, Danhong injection; MPO, myeloperoxidase. 

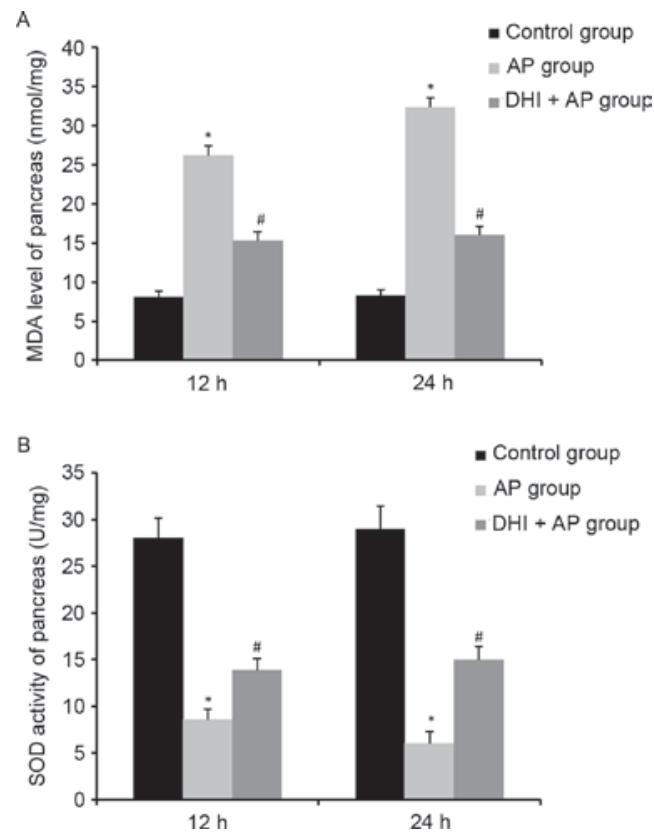

Figure 4. Effects of DHI on pancreatic injury in AP rats. (A) MDA level and (B) SOD activity. Data are presented as the mean \pm standard error ( $\mathrm{n}=10$ /group). ${ }^{\mathrm{P}}<0.01$ vs. control group; ${ }^{\text {}} \mathrm{P}<0.01$ vs. AP group. AP, acute pancreatitis; DHI, Danhong injection; MDA, malondiadelhyde; SOD, superoxide dismutase.
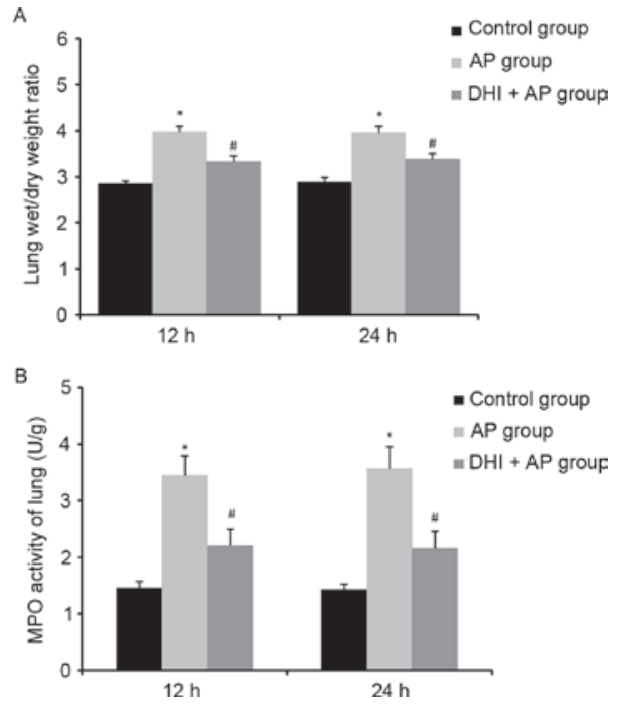

Figure 5. Effects of DHI on lung injury in AP rats. (A) Lung wet/dry weight ratio and (B) MPO activity. Data are presented as the mean \pm standard error ( $\mathrm{n}=10$ /group). ${ }^{*} \mathrm{P}<0.01$ vs. control group; ${ }^{\#} \mathrm{P}<0.01$ vs. AP group. AP, acute pancreatitis; DHI, Danhong injection; MPO, myeloperoxidase.

Statistical analysis. The data were analyzed by SPSS 18.0 (SPSS, Inc., Chicago, IL, USA). The results are expressed as the mean \pm standard error. The statistical significance was evaluated by one-way analysis of variance followed by Student-Newmane-Keuls test. $\mathrm{P}<0.05$ was considered to indicate a statistically significant difference.

\section{Results}

Histological examination of the effects of DHI on pancreatic and lung injury in AP. The model of AP was induced

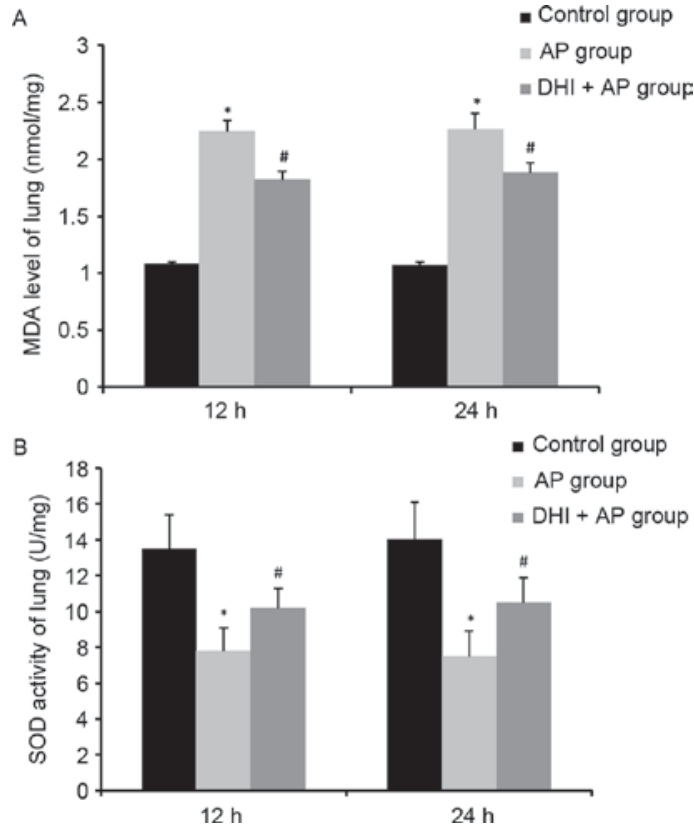

Figure 6. Effects of DHI on lung injury in AP rats. (A) MDA level and (B) SOD activity. Data are presented as the mean \pm standard error ( $n=10$ /group). ${ }^{*} \mathrm{P}<0.01$ vs. control group; ${ }^{\#} \mathrm{P}<0.01$ vs. AP group. AP, acute pancreatitis; DHI, Danhong injection; MDA, malondiadelhyde; SOD, superoxide dismutase.

by infusion of $5 \%$ sodium taurocholate $(1 \mathrm{ml} / \mathrm{kg})$. Compared with control rats (Fig. 1A), AP rats pancreas had significant morphological changes in the pancreas (Fig. 1B). Furthermore, an extensive infiltration of leukocytes into the pancreas, beside of tissue edema, blood vessel dilatation, and congestion vessel could be seen in the pancreas (Fig. 1B). Compared with the AP group, a significant reduction of acinar necrosis, edema, and inflammatory infiltration were observed in the DHI + AP group (Fig. 1C). No evident histological alteration was observed in the lung of control mice (Fig. 1D). AP resulted in significant lung injury, evidenced by presence of interstitial edema, alveolar thickening and extensive recruitment of neutrophils into the alveolar spaces (Fig. 1E). These pathological changes were improved by DHI administration (Fig. 1F). In both the lung (Fig. 2A) and the pancreas (Fig. 2B), AP significantly increased the histopathologic damage score; however, DHI treatment reduced this score. Therefore, DHI may prevent the pancreatic injury in AP.

$M P O$ activity, MDA levels and SOD activity in the pancreas in AP following DHI treatment. MPO activity, a marker of neutrophil function, is used for assessing the intensity of inflammation (23). In addition to increased plasma amylase levels (Fig. 3A), MPO activity (Fig. 3B) and MDA levels (Fig. 4A) were significantly enhanced in the AP group and markedly ameliorated by DHI. However, SOD activity was reduced by AP, but DHI significantly reversed this effect (Fig. 4B). These results suggested that DHI may reduce pancreatic inflammation.

Effect of DHI on lung wet/dry ratio in AP. Compared with the control group, the lung wet/dry ratio in the AP group increased significantly, whereas it was significantly attenuated in the 

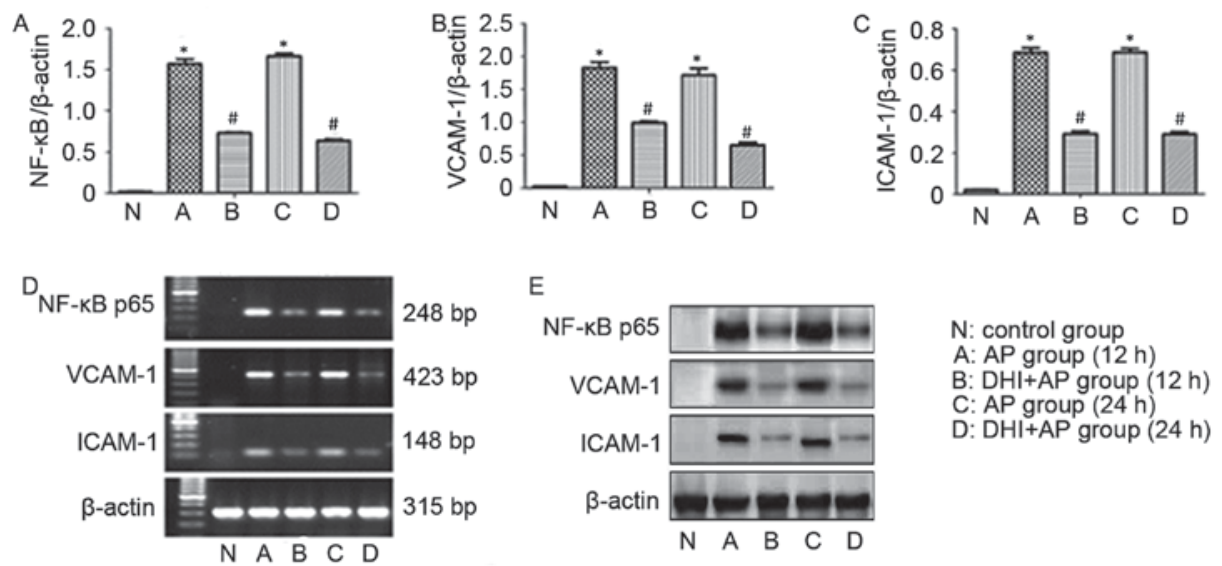

Figure 7. Effects of DHI on expression levels of VCAM-1, ICAM-1 and NF- $\kappa$ B p65 in AP rats. The mRNA expression levels of (A) NF- $\kappa$ B p65, (B) VCAM-1 and (C) ICAM-1. (D) RNA and (E) protein expression levels VCAM-1, ICAM-1, NF- $\kappa$ B p65. Data are presented as the mean \pm standard error (n=10/group). ${ }^{*} \mathrm{P}<0.01$ vs. control group, ${ }^{\#} \mathrm{P}<0.01$ vs. AP group. AP, acute pancreatitis; DHI, Danhong injection; VCAM-1, vascular cell adhesion protein 1; ICAM-1, intracellular adhesion molecule $1 ; \mathrm{NF}-\kappa \mathrm{B}$, nuclear factor- $\kappa \mathrm{B}$.

DHI + AP group (Fig. 5A). Therefore, DHI could suppress AP-induced lung edema.

MPO activity, MDA levels and SOD activity in the lung in AP following DHI treatment. The role of DHI on MPO activity, MDA content and SOD activity in lung tissues were investigated. A significant rise in lung MPO activity (Fig. 5B) and MDA level (Fig. 6A), and a decrease in lung SOD activity (Fig. 6B) were observed in the AP group, indicating neutrophil infiltration, membrane lipid peroxidation and superoxide toxicity in lung tissue as a result of AP, respectively. Treatment with DHI ameliorated this effect.

$m R N A$ and protein expression level alterations in the lung in AP following DHI treatment. In order to gain understanding into the action of DHI on AP-associated lung injury at the molecular level, the effects of DHI on the mRNA and protein expression levels of VCAM-1, ICAM-1 and NF- $\kappa$ B p65 were assessed by RT-sqPCR and western blotting, respectively. AP rats exhibited significantly increased mRNA expression levels of NF- $\kappa \mathrm{B}$ p65 (Fig. 7A), VCAM-1 (Fig. 7B) and ICAM-1 (Fig. 7C). The same effect was observed from RT-sqPCR (Fig. 7D). However, treatment with DHI significantly reversed this effect. Representative western blot images are presented in Fig. 7E.

\section{Discussion}

DHI, a popular herbal medicine in China, consists of Salvia miltiorrhiza and Carthamus tinctorius. As described previously, DHI has served a positive role on scavenging oxygen free radicals, preventing lipid peroxide, inhibiting inflammatory reaction and improving microcirculation. Furthermore, inflammatory release and the oxygen reaction serve an important role in the pathogenesis of AP-associated multi-organ complications. Therefore, the present study investigated the protective effect of DHI on AP-associated lung injury.

$\mathrm{NF}-\kappa \mathrm{B}$ is as a transcription factor which regulates various genes involved in inflammatory and immune responses (24). Current research has focused on the association between $\mathrm{NF}-\kappa \mathrm{B}$ and AP (25). NF- $\mathrm{B}$ modulates the expression of numerous genes, such as genes encoding for cytokines, adhesion molecules and enzymes, which serves a pivotal role in the initiation, promotion and progression of the inflammatory response (26). In turn, AP could upregulate $\mathrm{NF}-\kappa \mathrm{B}$ expression, exacerbating inflammation to AP $(27,28)$. In the present study, in the AP group, NF- $\kappa \mathrm{B}$ p65 was markedly increased, and the mRNA and protein expression levels of adhesion factors (VCAM-1 and ICAM-1) were increased. After animals were treated with DHI, the activation of NF- $\mathrm{B}$ p 65 was significantly inhibited, and the expression of VCAM-1 and ICAM-1 were markedly decreased. These results demonstrated that DHI could inhibit NF- $\mathrm{B}$ p65 activation, modulate the expression of VCAM-1 and ICAM-1, and suppress the inflammatory response in AP.

Oxidative stress had been demonstrated to serve a key role in causing tissue damage in AP. Abnormal generation of reactive oxygen species occurs during the course of pancreatitis, leading to pancreatic oxidative stress and even systemic oxidative stress (29,30). Furthermore, the MDA level, an important index of oxidative stress, could increase expression of NF- $\kappa \mathrm{B}$ (31). In the present study, a significant rise of MDA level was observed in the AP group, whereas this was decreased in the DHI + AP group. In addition, the activity of SOD, an endogenous free radical scavenging agent which can eliminate oxyradicals, was also examined. AP decreased SOD activity in pancreas and lung tissue, while SOD activities increased in mice treated with DHI, compared with those in the AP group. These results indicated the DHI could effectively attenuate oxidative stress injury in AP-associated lung injury.

In conclusion, the present study demonstrated the protective and therapeutic effects of DHI on AP-associated lung injury by suppressing the $\mathrm{NF}-\kappa \mathrm{B}$ activity, decreasing neutrophil infiltration and the oxidative stress level, and reducing the expression levels endodermis attachment proteins (VCAM-1 and ICAM-1). Therefore, DHI may represent a potential candidate for therapy of AP.

\section{Acknowledgements}

The present study was supported by the Program of Science and Technology Planning of Health and Family Planning 
Commission of Jiangxi Province (grant nos. 20155239 and 20161074) and the Program of Science and Technology Planning of Nanchang (grant no. 2012-CYH-SXHZ-YLWS-001).

\section{References}

1. Lund H, Tønnesen H, Tønnesen $\mathrm{MH}$ and Olsen O: Long-term recurrence and death rates after acute pancreatitis. Scand J Gastroentero 41: 234-238, 2006.

2. Akbarshahi H, Rosendahl AH, Westergren-Thorsson G and Andersson R: Acute lung injury in acute pancreatitis-awaiting the big leap. Resp Med 106: 1199-1210, 2012.

3. Bhatia M, Brady M, Shokuhi S, Christmas S, Neoptolemos JP and Slavin J: Inflammatory mediators in acute pancreatitis. J Pathol 190: 117-125, 2000.

4. Norman J: The role of cytokines in the pathogenesis of acute pancreatitis. Am J Surg 175: 76-83, 1998.

5. Lane JS, Todd KE, Gloor B, Chandler CF, Kau AW, Ashley SW and McFadden DW: Platelet activating factor antagonism reduces the systemic inflammatory response in a murine model of acute pancreatitis. J Surg Res 99: 365-370, 2001.

6. Shabanov VV, Sarbaeva NN and Milyakova MN: Generation of free oxygen radicals in the pathogenesis of experimental acute reflux pancreatitis. Bull Exp Biol Med 134: 26-27, 2002.

7. Eşrefoğlu M, Gül M, Ates B, Batçioğlu K and Selimoğlu MA: Antioxidative effect of melatonin, ascorbic acid and $\mathrm{N}$-acetylcysteine on caerulein-induced pancreatitis and associated liver injury in rats. World J Gastroentero 12: 259-264, 2006.

8. Lapidot T, Walker MD and Kanner J: Antioxidant and prooxidant effects of phenolics on pancreatic $\beta$-cells in vitro. J Agr Food Chem 50: 7220-7225, 2002.

9. Sun M, Zhang JJ, Shan JZ, Zhang H, Jin CY, Xu S and Wang YL: Clinical observation of Danhong Injection (herbal TCM product from Radix Salviae miltiorrhizae and Flos Carthami tinctorii) in the treatment of traumatic intracranial hematoma. Phytomedicine 16: 683-689, 2009.

10. He Y, Wan H, Du Y, Bie X, Zhao T, Fu W and Xing P: Protective effect of Danhong injection on cerebral ischemia-reperfusion injury in rats. J Ethnopharmacol 144: 387-394, 2012.

11. Gao LN, Cui YL, Wang QS and Wang SX: Amelioration of Danhong injection on the lipopolysaccharide-stimulated systemic acute inflammatory reaction via multi-target strategy. J Ethnopharmacol 149: 772-782, 2013.

12. Huang YM,Zhang ZJ, Guo AM,Pan LH and Li CE: Determination of protocatechuic aldehyde in Danhong injection by HPLC. Jie Fang Jun Yao Xue Xue Bao Bian Ji Bu 21: 467-469, 2005 (In Chinese).

13. Wang SM: Determination of water-solubility component in Danhong injection by HPLC. Shi Zhen Guo Yi Guo Yao Bian Ji Bu 17: 989-990, 2006 (In Chinese).

14. Liu X, Wu Z, Yang K, Ding $\mathrm{H}$ and Wu Y: Quantitative analysis combined with chromatographic fingerprint for comprehensive evaluation of Danhong injection using HPLC-DAD. J Pharm Biomed Anal 76: 70-74, 2013.

15. Wan LH, Chen J, Li L, Xiong WB and Zhou LM: Protective effects of Carthamus tinctorius injection on isoprenaline-induced myocardial injury in rats. Pharm Biol 49: 1204-1209, 2011.
16. Chen YH, Lin SJ, Ku HH, Shiao MS, Lin FY, Chen JW and Chen YL: Salvianolic acid B attenuates VCAM-1 and ICAM-1 expression in TNF-alpha-treated human aortic endothelial cells. J Cell Biochem 82: 512-521, 2001.

17. Chen ZQ, Hong L and Wang H: Effect of Danhong injection on platelet activation and inflammatory factors in patients of acute coronary syndrome after intervention therapy. Zhongguo Zhong Xi Yi Jie He Za Zhi 29: 692-694, 2009 (In Chinese).

18. Wang L, Zhang X, Liu L, Cui L, Yang R, Li M and Du W: Tanshinone II A down-regulates HMGB1, RAGE, TLR4, NF-карраB expression, ameliorates BBB permeability and endothelial cell function and protects rat brains against focal ischemia. Brain Res 1321: 143-151, 2010.

19. Perides G, van Acker GJ, Laukkarinen JM and Steer ML: Experimental acute biliary pancreatitis induced by retrograde infusion of bile acids into the mouse pancreatic duct. Nat Protoc 5: 335-341, 2010

20. Schmidt J, Rattner DW, Lewandrowski K, Compton CC, Mandavilli U, Knoefel WT and Warshaw AL: A better model of acute pancreatitis for evaluating therapy. Ann Surg 215: 44-56, 1992.

21. Tanino Y, Makita H, Miyamoto K, Betsuyaku T, Ohtsuka Y, Nishihira $\mathbf{J}$ and Nishimura $M$ : Role of macrophage migration inhibitory factor in bleomycin-induced lung injury and fibrosis in mice. Am J Physiol Lung Cell Mol Physiol 283: L156-L162, 2002.

22. Simms D, Cizdziel PE and Chomczynski P: TRIzol: A new reagent for optimal single-step isolation of RNA. Focus 15: 532-535, 1993.

23. Yoshida M, Yamada M, Sudo Y, Kojima T, Tomiyasu T, Yoshikawa N, Oda T and Yamada M: Myeloperoxidase anti-neutrophil cytoplasmic antibody affinity is associated with the formation of neutrophil extracellular traps in the kidney and vasculitis activity in myeloperoxidase anti-neutrophil cytoplasmic antibody-associated microscopic polyangiitis. Nephrology (Carlton) 21: 624-629, 2016.

24. Medzhitov R and Horng T: Transcriptional control of the inflammatory response. Nat Rev Immunol 9: 692-703, 2009.

25. Pooran N, Indaram A, Singh P and Bank S: Cytokines (IL-6, IL-8, TNF): Early and reliable predictors of severe acute pancreatitis. J Clin Gastroenterol 37: 263-266, 2003.

26. Boone DL, Lee EG, Libby S, Gibson PJ, Chien M, Chan F, Madonia M, Burkett PR and Ma A: Recent advances in understanding NF-карраB regulation. Inflamm Bowel Dis 8: 201-212, 2002.

27. Shi C, Zhao X, Lagergren A, Sigvardsson M, Wang X and Andersson R: Immune status and inflammatory response differ locally and systemically in severe acute pancreatitis. Scand J Gastroenterol 41: 472-480, 2006.

28. Meng Y, Ma QY, Kou XP and Xu J: Effect of resveratrol on activation of nuclear factor kappa-B and inflammatory factors in rat model of acute pancreatitis. World J Gastroenterol 11: 525-528, 2005.

29. Leung PS and Chan YC: Role of oxidative stress in pancreatic inflammation. Antioxid Redox Signal 11: 135-166, 2009.

30. Yu JH, Lim JW, Kim KH, Morio T and Kim H: NADPH oxidase and apoptosis in cerulein-stimulated pancreatic acinar AR42J cells. Free Radical Bio Med 39: 590-602, 2005.

31. Seo JY, Kim H, Seo JT and Kim KH: Oxidative stress induced cytokine production in isolated rat pancreatic acinar cells: Effects of small-molecule antioxidants. Pharmacology 64: 63-70, 2002. 